\title{
س
}

$>\mathrm{DE}$

$\stackrel{1=1}{\simeq}$ PÉDAGOGIE

\author{
Recherches en éducation
}

$181 \mid 2012$

Varia

\section{Pourquoi expérimenter une politique éducative ? Le repérage des jeunes en décrochage scolaire}

What is the point of experimenting an educational policy? The identification of early school leavers

Pierre-Yves Bernard et Christophe Michaut

\section{OpenEdition}

\section{Journals}

Édition électronique

URL : http://journals.openedition.org/rfp/3886

DOI : $10.4000 /$ rfp.3886

ISSN : 2105-2913

Éditeur

ENS Éditions

Édition imprimée

Date de publication : 31 décembre 2012

Pagination : 5-14

ISBN : 978-2-84788-405-0

ISSN : 0556-7807

\section{Référence électronique}

Pierre-Yves Bernard et Christophe Michaut, « Pourquoi expérimenter une politique éducative? Le repérage des jeunes en décrochage scolaire », Revue française de pédagogie [En ligne], 181 | 2012, mis en ligne le 17 juin 2015, consulté le 26 juillet 2020. URL : http://journals.openedition.org/rfp/3886 ; DOI : https://doi.org/10.4000/rfp.3886 


\section{Pourquoi expérimenter une politique éducative? Le repérage des jeunes en décrochage scolaire}

\section{Pierre-Yves Bernard et Christophe Michaut}

Le suivi des élèves sortant du système éducatif sans diplôme ou avec une faible qualification ne fait pas l'objet d'un traitement systématique par les institutions publiques. Pour assurer un suivi exhaustif, un logiciel identifiant le devenir des jeunes concernés a été localement expérimenté avant d'être diffusé sur le territoire national. L'article revient sur la genèse de ce dispositif et analyse les logiques qui ont présidé au choix de cette expérimentation. S'agissait-il d'expérimenter pour gagner en efficacité, pour transformer des pratiques jugées obsolètes ou pour asseoir une légitimité ? L'observation du déroulement de l'expérimentation et les dix-huit entretiens réalisés auprès des responsables et des conseillers chargés de la mise en œuvre du dispositif révèlent l'existence de plusieurs registres de justification.

Mots-clés (TESE) : administration de l'enseignement, abandon scolaire, méthode d'évaluation, politique en matière d'éducation.

Les politiques éducatives sont aujourd'hui largement orientées vers des finalités de transformation du monde scolaire. Les arguments selon lesquels le changement des systèmes éducatifs est indispensable sont bien connus, largement exposés par les responsables des politiques éducatives et composent une véritable rhétorique de la réforme. D'une part, le monde éducatif doit faire face à de nouveaux enjeux : économie de la connaissance, évaluation des résultats, problématique de l'équité. D'autre part, l'institution éducative, jugée trop rigide, doit s'adapter aux nouveaux besoins. Pour combattre l'inertie structurelle du système, il serait nécessaire de développer une nouvelle gouvernance des systèmes scolaires (Bouvier, 2007). Dans ce type de discours, la notion d'expérimentation est fréquemment convoquée pour instituer de nouvelles formes d'action publique (Gomel \& Serverin, 2011). On entend expérimentation au sens de dispositif limité dans le temps et dans l'espace (L'Horty \& Petit, 2011). À ce titre, elle présente une rupture par rapport à une conception égalitaire et continue de l'action publique. Elle permettrait de tester des solutions jusque-là laissées de côté, de donner de la flexibilité au système et de réguler l'action au plus près des réalités de terrain. En somme, elle constitue une figure-type des nouvelles formes d'action publique fondées sur l'évaluation de "ce qui marche ". L'expérimentation est à cet égard un instrument de l'evidence-based policy d'une manière générale. Sa mise en œuvre dans le domaine éducatif ne serait que la transposition de méthodes déjà éprouvées dans d'autres secteurs de l'intervention publique, comme la santé 


\section{Le cadrage institutionnel de l'expérimentation des politiques éducatives en France}

Dans un contexte institutionnel de forte centralisation administrative et de normalisation des pratiques et des programmes scolaires, il est a priori très difficile pour les professionnels de développer des innovations dans une visée d'expérimentation. Depuis le début des années quatre-vingt, l'administration reconnaît davantage les initiatives se démarquant du cadre institutionnel standard, à partir de trois sources:

- les établissements expérimentaux : depuis 1982, il est possible d'ouvrir des établissements publics susceptibles d'accueillir, sous des formes pédagogiques nouvelles, les élèves en rupture avec le système scolaire classique ;

- la possibilité de déroger aux textes nationaux : la loi d'orientation et de programme pour l'avenir de l'école du 23 avril 2005 introduit cette possibilité avec son article 34 : " la réalisation d'expérimentations, pour une durée maximum de cinq ans, portant sur l'enseignement des disciplines, l'interdisciplinarité, l'organisation pédagogique de la classe, de l'école ou de l'établissement, la coopération avec les partenaires du système éducatif... " ;

- un financement spécifique : le fonds d'expérimentation pour la jeunesse est créé par la loi du 1er décembre 2008. Ce fonds fonctionne sur le principe de l'appel à projets et finance des actions destinées aux jeunes de moins de 25 ans. L'expérimentation présentée dans le texte relève de ce financement.

(Slavin, 2002 ; Laurent, Baudry, Berriet-Solliec et al., 2009), et fournirait l'instrument d'une éducation fondée sur la preuve (evidence-based education) (Normand, 2006 ; Saussez \& Lessard, 2009).

L'expérimentation des politiques éducatives est inscrite institutionnellement à plusieurs niveaux : possibilité d'expérimenter des pratiques nouvelles dans les établissements, de créer des structures innovantes, de recevoir des financements spécifiques... (voir encadré). Cette légitimité institutionnelle s'inscrit dans un vaste processus de transformation de l'action publique visant une rationalisation de la décision politique, celle-ci ne devant plus se fonder sur la reproduction indéfinie des mêmes recettes, mais au contraire s'appuyer sur les résultats avérés de nouveaux dispositifs.

Toutefois, l'argumentation développée autour des expérimentations ne dit rien sur les choix d'innovations ou sur le processus de construction des solutions. Ce qui est expérimenté résulte nécessairement d'une multitude de décisions et de sélections dont on peut douter qu'elles placent le décideur politique en situation de choix rationnel entre plusieurs alternatives qui s'imposeraient par leur évidence. Par ailleurs, rien ne garantit que la mise en œuvre d'une politique s'appuie sur les résultats de l'expérimentation, quels qu'ils soient. II semble donc important d'interroger ce recours à l'expérimentation dans les politiques éducatives, pour en comprendre le sens et dépasser la vision techniciste que l'expérimentation donne. Quel principal objectif une expérimentation de politique éducative poursuit-elle : résoudre un problème sur la base des résultats tirés de l'expérimentation, transformer les pratiques des acteurs ou asseoir une légitimité institutionnelle?

Les politiques de traitement du décrochage scolaire illustrent parfaitement cette volonté d'orienter les décisions en s'appuyant sur une démarche expérimentale. Le fonds d'expérimentation pour la jeunesse finance à ce titre plusieurs opérations sur le décrochage scolaire. L'une d'entre elles vise formellement à concevoir et à développer un logiciel de repérage et de partage de données individuelles, au niveau d'une région, pour assurer le suivi des élèves et des apprentis en situation de décrochage. Ce partage de données s'effectue entre différentes structures publiques en charge de la jeunesse, notamment le ministère de l'Éducation nationale, les missions locales et la Région. Le principal objectif est d'identifier les élèves ou les apprentis sortant sans diplôme du système éducatif, ce que ne permettent pas jusque-là les systèmes d'information existants (Conseil de l'emploi, des revenus et de la cohésion sociale, 2008). Dans le cadre de l'évaluation de ce dispositif que nous avons réalisée, il s'agit d'identifier les logiques d'action à partir de l'observation des instances de l'expérimentation et d'entretiens $(\mathrm{N}=18)$. Ces derniers ont été réalisés auprès de professionnels impliqués dans le comité de pilotage et de responsables de bassin de formation chargés de la mise en œuvre du dispositif1. L'analyse de ces données fait apparaître la portée et les limites de différents registres de 
justification de l'expérimentation modélisés en trois grands types : modèles de l'expérimentation rationnelle, instrumentale et légitimante.

Après un bref historique de l'expérimentation, une première partie présentera le modèle d'expérimentation rationnelle, qui sert de référence à la conception du dispositif. Une deuxième partie sera consacrée à la façon dont les acteurs eux-mêmes, dans le discours tenu aux chercheurs, relativisent cette visée rationnelle de l'expérimentation. Ce discours inscrit fondamentalement l'expérimentation dans une perspective instrumentale, où s'affirme une volonté de renouvellement de l'action publique à partir d'un outil technique. Une troisième partie tentera d'interpréter l'échec de l'expérimentation qui s'est arrêtée au bout d'un an, en faisant l'hypothèse qu'il résulte d'un conflit de légitimité entre institutions, ce qui laisse entrevoir un troisième modèle, plus implicite, qualifié d'expérimentation légitimante.

\section{EXPÉRIMENTER POUR RÉSOUDRE UN PROBLÈME ?}

\section{L'histoire d'un échec}

L'expérimentation dont il est question est portée par les services d'une Mission générale d'insertion (MGI) de l'Éducation nationale qui a la responsabilité du suivi des jeunes sortant ou risquant de sortir du système éducatif sans diplôme. Ce suivi pose le problème du repérage de ces jeunes. Au niveau d'un établissement, il est difficile de faire la part entre les sortants qui changent d'établissement ou de voie de formation (sorties vers l'apprentissage par exemple) et ceux qui quittent le système éducatif sans avoir obtenu une certification. Un premier dispositif, lancé en 2006, avait permis de créer un système de partage d'informations entre les établissements publics de l'Éducation nationale d'une même région. L'expérimentation évaluée ici est une extension de ce dispositif, à une échelle plus ambitieuse, puisqu'il s'agit de créer un logiciel de partage d'informations entre différents réseaux du système éducatif (Éducation nationale, enseignement agricole, apprentissage) en y incluant les missions locales, le principal réseau d'insertion des jeunes. Ce dispositif informatique doit permettre de voir si un jeune sortant d'un établissement est pris en charge par une structure de formation.
L'expérimentation fait l'objet d'une convention de " coopération relative au suivi et à l'insertion professionnelle des jeunes sortant du système éducatif ", signée le 22 décembre 2009 entre les différents partenaires concernés : l'administration déconcentrée de l'Éducation nationale, la Région, les missions locales pour l'insertion des jeunes, la Protection judiciaire de la jeunesse et l'administration territoriale de l'Agriculture. Outre l'engagement plus ou moins affirmé de partager l'information sur les jeunes pris en charge par telle ou telle institution, ce texte formalise également la mise en place d'instances territoriales de concertation. Ces instances doivent se charger d'organiser le recensement et le repérage des sortants et d'analyser les besoins des jeunes identifiés.

L'expérimentation démarre en janvier 2010. Le comité de pilotage se met en place, des entreprises sont choisies pour la mise en œuvre technique du dispositif, les instances territoriales sont constituées. Toutefois le contexte national change rapidement. Un autre outil, le Suivi de l'orientation (SDO), est développé par la Direction générale de l'enseignement scolaire (DGESCO). La situation devient alors inédite : une concurrence entre une administration nationale et une administration déconcentrée pour imposer un outil dont la vocation est d'être appliqué au niveau national. La circulaire du 18 mars 2010 impose l'outil SDO comme solution technique pour le partage des informations entre les établissements. Si l'expérimentation est abondamment sollicitée dans cette circulaire - à propos du handicap, de la sécurité routière, du livret de compétences -, elle n'est plus de mise quand il s'agit de la lutte contre le décrochage scolaire. La transmission d'informations des établissements d'enseignement vers les missions locales n'est plus présentée comme un partage mais comme une obligation légale.

En janvier 2011, alors que le dispositif d'échange de données est opérationnel, l'administration nationale signifie au rectorat de l'académie concernée que le dossier ne sera pas présenté à la Commission nationale de l'informatique et des libertés (CNIL) qui doit accorder son autorisation pour l'échange de données personnelles. Le ministère a mis au point un autre outil technique d'interconnexion des données, dérivé du SDO, le Service interministériel d'échange de données (SIEI), et destiné à être mis en place à la rentrée 2011. L'expérimentation s'achève avant même d'avoir fonctionné. Pourquoi dès lors expérimenter quand ni l'évaluation ni la possibilité de généralisation n'ont d'importance? 


\section{Le modèle de l'expérimentation rationnelle}

En première analyse, l'expérimentation de l'action publique se fonde sur un ensemble de principes qui se rapportent à une vision rationalisatrice de l'action publique. La conception de l'expérimentation développée au plan institutionnel est très proche de celle que préconisent un certain nombre d'économistes dans le domaine de l'évaluation des politiques publiques, notamment par la mise en œuvre d'une évaluation aléatoire. Formalisée par Rubin (1974), cette évaluation repose sur l'assignation aléatoire d'un groupe expérimental recevant le traitement et d'un groupe contrôle ne le recevant pas. La comparaison des deux groupes à l'issue de l'expérimentation permet ainsi une mesure non biaisée des effets du traitement. Cette méthode est souvent considérée comme la mieux à même de mesurer les effets d'une action publique, afin d'éviter les biais de sélection, comparativement aux méthodes quasi expérimentales ou aux modélisations économétriques (L'Horty \& Petit, 2011). Par ailleurs, des actions innovantes en matière éducative se sont appuyées dans certains pays sur des travaux d'évaluation à valeur scientifique, plutôt en provenance du champ de l'économie. L'expérimentation, à condition qu'elle réponde à certains critères, notamment de choix des groupes test et témoin, est censée garantir la scientificité de l'évaluation.

On a donc un modèle d'action, appelé ici modèle de l'Expérimentation rationnelle (ER), qui peut se définir de manière séquentielle selon le schéma suivant. Un problème politique se pose, qui traduit une déficience des pouvoirs publics à y faire face. II faut donc développer une innovation pour répondre au problème posé. Pour s'assurer des effets de cette innovation, une expérimentation comportant un groupe de bénéficiaires et un groupe de non-bénéficiaires est mise en œuvre. Une évaluation scientifique des effets permet de vérifier si l'innovation répond ou non aux objectifs assignés. Éclairés par les résultats ainsi obtenus, les pouvoirs publics décident ou non de généraliser l'expérimentation². Cette modélisation fait de l'action publique une démarche qui se veut scientifique. On retrouve ici la manifestation d'un projet visant à transformer les questions politiques en questions d'expertise (Birnbaum, 1975).

Dans ce modèle, une place ambigüe est tenue par une figure importante, celle du porteur de projet. Si on suit le modèle ER, c'est un entrepreneur au sens schumpétérien du terme : il assure la dynamique du système politique par l'innovation, à la fois en créant de nouveaux dispositifs et en participant à la disparition des structures anciennes. Le système a donc besoin d'innovateurs ; mais selon quels mobiles agissent-ils ? Si l'on s'en tient au modèle économique, c'est le gain, ou plutôt l'espoir de gain, qui incite à l'innovation. Or une innovation de l'action publique n'est pas censée produire des gains privés, mais, par définition, des gains publics, par exemple la réduction des coûts sociaux des conséquences du décrochage scolaire. Une manière de sortir de ce paradoxe est de considérer que le gain recherché se définit en termes de pouvoir ou de légitimité, comme on le verra dans la troisième section de ce texte. Mais alors, on sort du modèle de la décision rationnelle où l'impulsion première de l'innovation repose sur son pouvoir technique de résoudre un problème défini a priori.

L'analyse des entretiens recueillis auprès des responsables locaux révèle une absence totale d'adhésion à ce modèle de l'expérimentation rationnelle. En toute franchise, ils déclarent qu'une expérimentation n'a pas pour finalité d'être évaluée pour participer à une prise de décision publique. Ce scepticisme vis-à-vis du modèle « rationnel " ne signifie pas un manque total d'adhésion à l'expérimentation. Ce qui compte en réalité, c'est le contenu de l'expérimentation et l'orientation qu'elle donne à la politique éducative : "Moi, je pense que c'est ça, l'expérimentation. [...] ça re-cimente des collaborations. Il y a un effet... il y a un effet moteur, quand même, hein, sans être dupe de tout ce qu'il y a. Mais c'est vrai qu'on va tous s'agiter un petit peu autour de ça. [...] ça va nous redonner de l'élan ; ça, c'est certain. Après, la durée de vie... Ben, on sait bien. Je veux dire, on n'est pas dupe. " (directrice d'un centre d'information et d'orientation).

C'est au niveau de l'orientation politique que les interlocuteurs se positionnent, dans la justification ou la critique du dispositif. Alors que pour beaucoup, il s'agit d'impulser ou de relancer une véritable coopération interinstitutionnelle, particulièrement entre l'Éducation nationale et les missions locales, certains voient dans l'outil un instrument de contrôle et de standardisation qui éloigne leur pratique de ce qu'ils estiment être leur mission : "On échappera au formatage informatique, j'espère, longtemps. Tant qu'on continuera à assurer des entretiens. " (directrice d'un centre d'information et d'orientation).

\section{EXPÉRIMENTER POUR TRANSFORMER L'ACTION PUBLIQUE ?}

\section{Le modèle de l'expérimentation instrumentale}

En référence aux travaux de Lascoumes et Le Galès qui considèrent l'instrumentation de l'action publique comme «l'ensemble des problèmes posés par le choix 
et l'usage des outils (des techniques, des moyens d'opérer, des dispositifs) qui permettent de matérialiser et d'opérationnaliser l'action gouvernementale » (2005, p. 12), le modèle de l'expérimentation instrumentale dépasse la seule fonction technique pour se focaliser sur le sens politique que les acteurs lui attribuent. Appliquée à la présente expérimentation, la gestion du dispositif révèle les différents niveaux caractéristiques (Tripier, 2003) : un substrat technique (un logiciel de partage de données), une représentation schématique de l'organisation (le partenariat interinstitutionnel) et une philosophie gestionnaire (le repérage et le suivi individualisé des jeunes).

II est frappant de constater combien le substrat technique a peu de valeur aux yeux des personnes impliquées dans le partenariat. Non pas que la technicité de l'outil et sa capacité à résoudre les problèmes soient ignorées, mais cette dimension technique est finalement reportée sur les experts mobilisés pour l'occasion: développeur informatique travaillant pour le rectorat, entreprise d'assistance à maîtrise d'ouvrage, entreprise d'ingénierie informatique.

Il faut également remarquer que la catégorisation utilisée, "jeune sortant du système éducatif sans solution ", est loin de réunir en une communauté de sens les partenaires de cette action publique. II y a là des cultures professionnelles, des référentiels d'action publique et des postures stratégiques très différents produisant engagement ou désengagement, alliance ou distanciation entre institutions. Le jeune " sans solution " est d'abord le produit d'une catégorisation scolaire, déplaçant l'attention des politiques publiques pour la jeunesse vers les sorties "problématiques" du système éducatif. De ce fait, les réserves sur cette catégorisation émanent des acteurs les moins impliqués dans le dispositif : "Aujourd'hui, on parle beaucoup du décrochage. Moi, j'ai horreur de ce terme-là. Nous, on parle de "rupturants" [des contrats d'apprentissage]. Et puis là, il y a une ambiguïté entre "jeunes sans solutions", "jeunes décrocheurs". " (responsable du service éducation de la Région). «Pour nous, le décrocheur, il est celui qui a décroché avant 16 ans. Pour l'Éducation nationale, il est celui qui a un parcours chaotique à partir de 16 ans. Donc on voit bien qu'on n'est pas du tout sur le même regard porté. " (conseillère de la Protection judiciaire de la jeunesse).

En fait, la dimension qui semble susciter le plus l'attention des acteurs se situe sur le registre du partenariat. Pour les acteurs de terrain, c'est l'opportunité de penser une meilleure articulation de l'action entre services différents, voire d'institutionnaliser des pratiques personnelles initiées localement. Pour les promoteurs de l'innovation, il s'agit d'impulser une dynamique, par les apports mutuels que permet la synergie du dispositif. À ce titre, la question du repérage n'est pas anodine. Elle se situe dans une problématique plus large du partage des informations entre partenaires, et des effets de ce partage en termes de régulation et d'apprentissage. On retrouve dans cette expérimentation la fonction d'apprentissage-transaction, qui permet de " caractériser les pratiques qu'engagent les individus et les groupes dans l'ensemble quotidien de leurs ajustements réciproques " (Gaudin, 2007, p. 187). Enfin, il est possible que l'outil soit un instrument de transformation systémique. Certains acteurs y voient des perspectives ambitieuses, notamment dans le projet de création d'un véritable service public de l'orientation, restructuré sur une base régionale. D'une manière plus générale, et c'est à ce niveau que l'expérimentation a un sens, il s'agit de partir du local pour transformer l'action publique au niveau national : "L'outil [...] c'est une anticipation régionale à la problématique nationale. C'est-à-dire que la Région est partie un an, deux ans avant la circulaire nationale. " (responsable à la Direction régionale de l'alimentation, de l'agriculture et de la forêt).

Se dessine dans ce modèle de l'expérimentation instrumentale une tout autre conception des finalités de l'expérimentation. Celle-ci n'est pas une procédure quasi scientifique d'aide à la décision, mais un outil en lui-même porteur de sens. Plus précisément, il s'agit d'un instrument visant la transformation des pratiques en matière d'action publique. II a pour objectif de remettre en cause les structures considérées comme anciennes, rigides et inadaptées, pour doter les décideurs d'outils de «nouvelle gouvernance ». En tant qu'expérimentation, elle permet de «tester " non pas ses effets supposés, mais sa capacité à transformer le système. En partant des initiatives locales, elle constitue une opportunité de court-circuiter les hiérarchies fonctionnelles habituelles, pour rapprocher les pratiques des besoins tels qu'ils s'expriment sur le terrain, au nom d'un registre de la proximité (Berthet, 2007). Assimilable à un "ballon d'essai ", l'évaluation de l'expérimentation porte plus sur sa réception que sur ses effets.

\section{Une illusion rhétorique?}

$\mathrm{Ni}$ le modèle de l'expérimentation rationnelle, ni le modèle de l'expérimentation instrumentale ne 
permettent de rendre compte du déroulé et de la fin du dispositif. Serait-on alors face à une illusion rhétorique ? Les pratiques réelles seraient-elles totalement déconnectées d'un discours qui n'aurait pour fonction que de les cacher sous une apparente modernité ? L'observation que nous avons réalisée ne nous permet pas de tirer une telle conclusion. II est toutefois important de s'arrêter sur cet aspect rhétorique, parce qu'il met en évidence une dimension importante du processus politique, celle de la légitimité.

Suivant la logique du premier modèle, la décision d'arrêter au dernier moment une expérimentation, qui aurait pu donner de précieuses indications sur les effets du repérage sur le décrochage scolaire, paraît surprenante. La seule justification de l'arrêt de l'expérimentation serait dans ce modèle un résultat d'évaluation mettant en évidence une absence d'effets, ou des effets inattendus et négatifs sur le problème posé. Selon le second modèle, l'arrêt de l'expérimentation avant son évaluation est beaucoup plus compréhensible : celle-ci peut en particulier manifester l'importance des résistances structurelles du système face aux perspectives de changement qu'apporte le nouveau projet. En réalité, l'interruption du dispositif ne se situe pas dans ce cas de figure. L'expérimentation est arrêtée, mais elle est généralisée à la France entière, avec toutefois un outil différent, mais qui repose sur le même principe : le partage des données entre Éducation nationale, ministère de l'Agriculture, Régions et missions locales.

Cette situation paradoxale ouvre la voie à une critique radicale de la notion d'expérimentation dans le domaine des politiques éducatives, du moins dans l'usage qui est fait de ce terme en France. Elle pourrait être considérée comme une pure figure de rhétorique. D'un côté, l'expérimentation consisterait à créer et à diffuser une représentation du problème pour légitimer telle ou telle forme d'action. Le modèle de l'expérimentation rationnelle serait alors une justification de décisions politiques déjà prises. Au-delà du lancement de l'expérimentation, qui peut ne constituer qu'une "vitrine ", la faiblesse, voire l'absence de suivi fréquemment observées (Gaudin, 2007) éviteraient de se poser la question de la réalité des effets attendus.

D'un autre côté, l'expérimentation peut chercher à masquer la réalité d'un exercice top-down du pouvoir, au niveau local comme dans l'articulation national/ régional. Les entretiens révèlent sur ce point une forte permanence de la structure verticale du pouvoir dans l'administration française, particulièrement au sein de l'Éducation nationale. Un élément qui semble aller dans le sens d'une inconsistance de l'expérimentation est la faible mobilisation des acteurs pour délimiter les enjeux centraux de l'action (la catégorisation des jeunes en situation de décrochage, les finalités du suivi individualisé et plus généralement les finalités du système éducatif), au détriment de la discussion sur les moyens mis en œuvre. Tout se passe comme si on laissait le terrain discuter des procédures et des moyens, alors que les orientations fondamentales restaient l'apanage du pouvoir central.

Pourtant, le dévoilement consistant à faire apparaître du pur discours là où on est censé trouver de l'action semble peu satisfaisant. Tout d'abord le comportement des acteurs, réellement mobilisés pendant la période d'expérimentation, peut difficilement être analysé sous l'angle de la manipulation, sauf à les voir comme des « idiots culturels". Ensuite, la décision d'arrêter l'expérimentation a suscité chez les acteurs impliqués au niveau local une réaction, certes mesurée, mais malgré tout explicite visant à la poursuite de l'expérimentation. Le porteur du projet a argumenté pour le défendre auprès du ministère et surtout permettre une mise en œuvre effective malgré le refus par la DGESCO du dépôt du dossier auprès de la CNIL.

\section{EXPÉRIMENTER POUR DEVENIR LÉGITIME}

\section{Le modèle de l'expérimentation légitimante}

Ces derniers évènements montrent l'existence de conflits au sein même de l'administration de l'Éducation nationale, conflits qu'il serait illusoire de réduire à des contingences locales ou personnelles. II est plus fécond d'éclairer ces désaccords à partir des notions de légitimité et d'intérêt général, en référence aux modèles d'action qui considèrent que toute forme de justification de l'action s'appuie sur une conception du bien commun. En ce sens, la légitimité, c'est-àdire la reconnaissance selon un principe de justice, mobilise une conception de l'intérêt général. Selon cette approche, il existe une pluralité des conceptions du bien commun, ce qui ouvre au conflit, à la critique et au compromis (Boltanski \& Thévenot, 1991). Si dans le champ de l'action publique l'intérêt général peut être vu comme un « ensemble de croyances dont l'efficacité dépend des relations entre la légitimation de l'action publique et la restructuration périodique des pouvoirs " (Gaudin, 2007, p. 59), on peut dès lors considérer que plusieurs "ensembles de croyances" se sont confrontés à l'occasion de cette expérimentation, et 
que celle-ci visait finalement une « restructuration des pouvoirs » qui n'a pas abouti.

En matière d'éducation, les principes de justice et de bien commun peuvent être modélisés à partir de conventions éducatives distinctes, comme autant de répertoires d'action qui organisent les finalités des activités éducatives, les pratiques pédagogiques et la structuration des institutions éducatives (Verdier, 2008). Dans une convention académique, la compétition scolaire entre les individus et la rupture supposée entre le monde scolaire et les appartenances sociales et locales des personnes amènent à donner à l'État une place centrale, notamment comme garant de l'équité du système éducatif. Dans d'autres conventions, universaliste ou professionnelle notamment, une structuration juste de l'institution scolaire impose que celle-ci soit profondément ancrée dans la société, ce qui ouvre notamment la possibilité d'une forte inscription dans des configurations territoriales.

À cet égard, la question du décrochage scolaire est révélatrice de la crise profonde que rencontre la convention académique dominante dans le système éducatif français (Bernard, 2011). Les premières années du $x x^{e}$ siècle ont vu l'ouverture d'une fenêtre d'opportunités mettant le traitement du décrochage scolaire à l'ordre du jour politique : prescription de la Commission européenne dans le cadre du processus de Lisbonne, relance des objectifs de certification à travers la loi d'orientation de 2005 , relance de la capacité d'initiative dans le domaine de la jeunesse avec le haut-commissariat à la Jeunesse créé en 2009.

L'expérimentation, parce qu'elle rompt l'unité institutionnelle du système éducatif national, met à mal la convention académique qui repose sur une conception uniforme de l'enseignement au niveau national. À l'inverse, les principaux protagonistes de l'expérimentation étudiée privilégient une prise en charge locale qui intègre un objectif d'insertion sociale et professionnelle. Les missions locales se sont historiquement constituées comme une voie de recours pour les jeunes non qualifiés par la voie scolaire, et visent l'accès à la qualification par des voies spécifiques. Les services rectoraux de la MGI, bien que services d'État, sont fortement marqués par une inscription territoriale et par des formes pédagogiques (accompagnement individualisé, parcours « à la carte ») plutôt en opposition avec la forme scolaire dominante. Les services éducatifs de la Région, enfin, sont également dans une posture assez éloignée de celle du monde scolaire académique. En charge des contrats d'apprentissage, ils côtoient régulièrement le monde professionnel et ont surtout pour but de " permettre à tout jeune d'avoir une solution de formation ou d'insertion professionnelle " (responsable du service éducation de la Région). Les acteurs précédemment cités se rapprochent finalement d'une convention universaliste selon laquelle l'essentiel est de permettre au plus grand nombre d'accéder à des savoirs en leur offrant une seconde chance, ou s'inscrivent dans une convention professionnelle qui vise à aider les jeunes dans leur insertion professionnelle et sociale par une reconnaissance de leurs compétences.

Par ailleurs, le fait que l'expérimentation soit financée par un fonds d'expérimentation sous tutelle de la direction de la Jeunesse, de l'Éducation populaire et de la Vie associative n'est pas sans signification. Cette administration de la jeunesse s'est constituée historiquement en se démarquant de l'administration de l'Éducation nationale, notamment en portant les valeurs de l'éducation populaire, en opposition aux principes académiques dominants dans le système éducatif (Prost, 2004). C'est donc le lieu institutionnel idéal pour lancer des innovations, des expériences qui vont dans le sens d'une politique de lutte contre les inégalités scolaires, pour la transformation des structures du système éducatif, en contournant l'appareil du ministère de l'Éducation nationale.

Finalement, l'expérimentation n'est pas seulement instrumentale, elle est légitimante. Le modèle le plus à même de rendre compte de l'histoire particulière de ce dispositif est un modèle où le sens de l'expérimentation réside principalement dans la légitimité des acteurs qui la porte. L'expérimentation est dès lors profondément politique, puisqu'elle ne vise pas seulement à transformer l'orientation de l'action publique, comme dans le modèle instrumental, elle participe d'une restructuration des centres de décision légitimes dans le système éducatif (Maroy \& Mangez, 2008). Dans le modèle légitimant, l'expérimentation participe pleinement à la construction du problème, comme mise en cause des principes jusque-là établis. L'expérimentation fonctionne comme un " point de passage obligé ", permettant la traduction d'un problème en dispositif (Callon, 1986).

\section{Une reprise en main du centre}

Il y a toutefois un paradoxe dans la volonté de transformation affichée. Elle s'appuie fortement sur une vision rationalisatrice de la politique éducative. La volonté d'expérimentation n'est pas porteuse d'un projet pédagogique spécifique, qui puisse démarquer ses promoteurs du modèle dominant de l'Éducation nationale, pourtant par ailleurs décrié. Cette orientation 
est la principale faiblesse de l'expérimentation : s'il s'agit de développer une application informatique permettant de repérer l'ensemble des sortants sans diplôme, quel acteur est mieux placé que l'administration de l'Éducation nationale ? À supposer que la recherche de légitimité soit à l'origine d'une expérimentation, la pérennité de celle-ci demeure fortement dépendante de l'environnement institutionnel. Très rapidement le porteur du projet prend conscience des limites de l'expérimentation: " [Le logiciel] a vécu une empathie complète quand je l'ai présenté au niveau national. C'était quelque chose d'assez remarquable pour eux, d'avoir réussi à faire ce travail-là. Et puis, tout de suite, ce n'est plus quelque chose qui devient intéressant parce qu'il faut qu'ils créent leur outil. [Le logiciel] est vite devenu quelque chose qu'il fallait oublier pour être remplacé par un système qu'ils voulaient faire eux-mêmes. » (responsable du projet).

Le départ de Martin Hirsch et l'intégration de la direction de la Jeunesse au sein du ministère de l'Éducation nationale signent la reprise en main de la politique éducative par l'Éducation nationale, et plus particulièrement par sa direction centrale (DEGSCO). Dans ce contexte, laisser poursuivre une expérimentation locale n'a plus de sens. L'administration centrale reprend la main en rendant obligatoires la constitution de partenariats au niveau local et le partage des données entre administrations. En «sifflant la fin du match », pour reprendre l'expression d'un de nos interlocuteurs, la DGESCO réaffirme la légitimité de l'État dans la conduite de la politique éducative. On pourrait objecter qu'en promouvant un outil de repérage du décrochage scolaire, l'État prend acte d'une transformation profonde du système éducatif, dans le sens d'une plus grande attention portée aux jeunes les plus disqualifiés par le système scolaire et d'un renforcement des partenariats entre acteurs publics.

On peut cependant rester sceptique sur l'issue d'un traitement centralisé du décrochage scolaire. Les expériences passées de la délégation interministérielle à l'insertion des jeunes et des réseaux publics pour l'insertion des jeunes ont largement montré les limites d'un partenariat local prescrit par une administration centrale. L'inertie institutionnelle, l'importance réaffirmée des principes de la convention académique (l'excellence et le mérite), l'opacité importante des décisions prises au sein du ministère, mais aussi et surtout l'oubli du public que révèle une action limitée au seul repérage augurent mal des effets des nouvelles mesures mises en place.

\section{CONCLUSION}

L'étude présentée a finalement déplacé la question initiale. La question "pourquoi expérimenter une politique éducative ? ", à laquelle il est possible de répondre selon le modèle de référence choisi, pour tester ses effets (modèle de l'expérimentation rationnelle) ou pour tester sa réception (modèle de l'expérimentation instrumentale), nous a conduits à poser la question "pourquoi des acteurs expérimentent-ils une politique éducative ? ", dans un modèle de l'expérimentation légitimante, qui amène à s'interroger sur les recompositions plus structurelles visées par une innovation. Ces modèles constituent en eux-mêmes des répertoires argumentatifs visant à justifier l'usage de l'expérimentation : on voit notamment que le modèle rationnel est fréquemment sollicité dès lors que l'expérimentation est inscrite institutionnellement dans les textes, alors même que ce modèle n'est pas mobilisé par les acteurs de terrain, qui s'accaparent plutôt l'expérimentation dans le cadre d'un modèle instrumental. Le modèle légitimant, quant à lui, est celui qui place l'expérimentation au cœur des choix de politique éducative, au sens de la légitimité des initiatives à l'origine des expérimentations. Mais ce sont également des modèles analytiques. Après tout, le modèle rationnel reste pertinent dès lors qu'on s'interroge sur les effets d'une expérimentation. Les modèles instrumentaux et légitimants permettent de comprendre les orientations recherchées par les expérimentations, et les enjeux de pouvoir qu'elles suscitent. Le dispositif évalué ici montre que le recours à ces deux modèles est indispensable pour en comprendre l'échec. On y décèle une orientation des politiques de traitement du décrochage scolaire, à la fois vers la standardisation des procédures et la personnalisation des actions, orientation profondément en tension avec la recherche de légitimité des acteurs portant l'expérimentation, tension résolue ici par un retour de la centralisation de la politique éducative.

Ce texte est tout particulièrement dédié à la mémoire de Franck Sanselme, trop tôt disparu, qui a participé à l'évaluation analysée ici.

Pierre-Yves Bernard pierre-yves.bernard@univ-nantes.fr Université de Nantes, Centre de recherche en éducation de Nantes

Christophe Michaut christophe.michaut@univ-nantes.fr Université de Nantes, Centre de recherche en éducation de Nantes 


\section{NOTES}

1 Liste des professionnels interviewés : un responsable de la Protection judiciaire de la jeunesse (PJJ), un responsable de la Direction régionale de la jeunesse, des sports et de la cohésion sociale (DRJSCS), un responsable de la Direction régionale de l'alimentation, de l'agriculture et de la forêt (DRAAF), un responsable de la Direction régionale de Pôle emploi, un attaché à la Direction régionale du travail, de l'emploi et de la formation professionnelle (DRTEFP), un responsable national des missions locales et deux responsables de bassin, un responsable au Conseil régional, un responsable académique et deux coordinateurs départementaux de la Mission générale d'insertion de l'Éducation nationale (MGIEN), cinq directeurs de Centre d'information et d'orientation (CIO), et un inspecteur de l'Éducation nationale Information-Orientation.

2 Dans le champ éducatif, la démarche du dispositif « La mallette des parents » illustre cette méthode d'évaluation (Avvisati, Gurgand, Guyon et al., 2010).

\section{BIBLIOGRAPHIE}

AVVISATI F., GURGAND M., GUYON N. \& MAURIN É. (2010). Quels effets attendre d'une politique d'implication des parents d'élèves dans les collèges ? Les enseignements d'une expérimentation contrôlée. Rapport pour le hautcommissaire à la Jeunesse. Paris : École d'économie de Paris.

BERNARD P.-Y. (2009). La politique de traitement du décrochage scolaire. Le cas de la Mission générale d'insertion de l'Éducation nationale. Thèse de doctorat, sciences de l'éducation, université de Nantes.

BERNARD P.-Y. (2011). Le décrochage scolaire. Paris : PUF.

BIRNBAUM P. (1975). La fin du politique. Paris : Éd. du Seuil.

BERTHET T. (2007). " L'État social à l'épreuve de l'action territoriale ". In A. Faure \& E. Négrier, Les politiques publiques à l'épreuve de l'action locale. Paris : L'Harmattan, p. 43-51.

BOLTANSKI L. \& THÉVENOT L. (1991). De la justification. Les économies de la grandeur. Paris: Gallimard.

BOUVIER A. (2007). La gouvernance des systèmes éducatifs. Paris : PUF.

CALLON M. (1986). "Éléments pour une sociologie de la traduction. La domestication des coquilles SaintJacques et des marins pêcheurs dans la baie de SaintBrieuc ». L'Année sociologique, vol. 36, p. 169-208.

CONSEIL DE L'EMPLOI, DES REVENUS ET DE LA COHÉSION SOCIALE (2008). Un devoir national. L'insertion des jeunes sans diplôme. Paris : La Documentation française.

GAUDIN J.-P. (2007). Gouverner par contrat. Paris : Presses de Science Po.

GOMEL B. \& SERVERIN É. (2011). Évaluer l'expérimentation sociale. Paris : Centre d'études de l'emploi. En ligne : http://www.cee-recherche.fr/fr/doctrav/143-evaluerexperimentation-sociale.pdf (consulté le 2 décembre 2011).

HIRSCH M. (2005). Au possible nous sommes tenus. La nouvelle équation sociale. Paris : Ministère des Solidarités, de la Santé et de la Famille.
LASCOUMES P. \& LE GALÈS P. (2005). Gouverner par les instruments. Paris : Presses de Science Po.

LAURENT C., BAUDRY J., BERRIET-SOLLIEC M. et al. (2009). "Pourquoi s'intéresser à la notion d'"evidencebased policy" ? ". Revue Tiers Monde, no 200, p. 853873.

L'HORTY Y. \& PETIT P. (2011). "Évaluation aléatoire et expérimentations sociales ". Revue française d'économie, vol. XXVI, n० 1, p. 13-48.

MAROY. C. \& MANGEZ C. (2008). "Rationalisation de l'action publique ou politisation de la connaissance ? ". Revue française de pédagogie, no 164, p. 87-90.

MAURIN É. (2007). La nouvelle question scolaire: les bénéfices de la démocratisation. Paris : Éd. du Seuil.

NORMAND R. (2006). " Les qualités de la recherche ou les enjeux du travail de la preuve en éducation ". Éducation et sociétés, no 18, p. 73-91.

PROST A. (2004). Histoire générale de l'enseignement et de l'éducation en France, tome IV : I'École et la famille dans une société en mutation. Depuis 1930. Paris : Perrin.

RUBIN D. B. (1974). "Estimating Causal Effects of Treatments in Randomized and Non-randomized Studies ». Journal of Educational Psychology, vol. 66, p. 688-701.

SAUSSEZ F. \& LESSARD C. (2009). "Entre orthodoxie et pluralisme, les enjeux de l'éducation basée sur la preuve ". Revue française de pédagogie, no 168, p. 111136.

SLAVIN R. E. (2002). " Evidence-Based Education Policies: Transforming Educational Practice and Research". Educational Researcher, vol. 31, no 7, p. 15-21.

TRIPIER P. (2003). "La sociologie des dispositifs de gestion : une sociologie du travail ». In V. Boussard \& S. Maugeri, Du politique dans les organisations. Paris : L'Harmattan, p. 11-24.

VERDIER É. (2008). " L'éducation et la formation tout au long de la vie : une orientation européenne, des régimes d'action publique et des modèles nationaux en évolution ". Sociologie et sociétés, vol. 40, n 1 , p. $195-225$ 